\title{
STELLAR ROTATION AND MIXING
}

\author{
JEAN-PAUL ZAHN \\ Département d'Astrophysique Stellaire et Galactique, \\ Observatoire de Paris, 92195 Meudon, France
}

\begin{abstract}
Many observations indicate that some mixing occurs in stellar radiation zones: in massive stars, chemical elements resulting from nuclear burning in the core are detected at the surface, and in solar-type stars lithium is depleted with age. Since all mixing processes transport also momentum, the depletion of lithium should be linked with the loss of angular momentum through the stellar wind, and there are indeed signs of such a correlation in the behavior of tidally-locked binaries. Moreover, any transport process leaves its signature in the internal rotation profile, and this can help greatly in its identification. After reviewing the main transport mechanisms which have been considered so far, our present conclusion is that the uniform rotation observed in the radiative interior of the Sun is probably achieved by the action of internal waves emitted at the base of the convective envelope. It remains to be verified whether these waves contribute directly to the mixing, or whether they act only through the shaping of the rotation profile, which in turn determines the mixing through meridian circulation and turbulent diffusivity.
\end{abstract}

\section{Mixing in stellar interiors: the observational evidence}

At first sight, it seems that stars undergo no mixing at all during their main sequence phase, since most of them evolve towards the giant branch, which proves that they build up a helium rich core surrounded by a hydrogen rich envelope. This heterogeneous structure arises because a fraction of the stellar interior is in radiative equilibrium - if the star were fully convective, it would remain homogeneous.

At a closer look however, many stars show some signs of partial mixing occurring even in their radiative regions, and we shall briefly recall them. 
Chemical elements which are produced through nuclear reactions in the innermost regions of stars are observed at their surface, such as ${ }^{14} \mathrm{~N}$ in $\mathrm{B}$ stars (Lyubimkov 1984, 1991; Gies \& Lambert 1992). An enhancement of ${ }^{4} \mathrm{He}$ is detected in some OB stars (Herrero et al. 1992), and there is an excess of ${ }^{13} \mathrm{C}$ in RGB stars (Lambert 1976). Likewise, the overabundance of ${ }^{3} \mathrm{He}$ in the solar wind suggests that this isotope originates from the deep interior of the Sun (Geiss \& Reeves 1972).

On the other hand, the surface abundance of ${ }^{7} \mathrm{Li}$ decreases with age in solar-type stars (Herbig 1965; Soderblom et al. 1993), which impli that this fragile element has been carried some distance below the convective envelope, to be destroyed where the temperature exceeds $\approx 2.5,10^{6} \mathrm{~K}$.

There is also indirect evidence that some mild mixing occurs in stars possessing a radiative envelope; otherwise, as was pointed out by Biermann (1937) and again by Schatzman (1969), microscopic processes such as atomic diffusion, gravitational settling and radiative acceleration would cause significant differences in their surface composition, which are not observed as a rule.

The purpose of this contribution is to examine the possible causes of such mixing, and to verify whether it is related to the rotation of the stars.

\section{Rotational mixing in early-type stars}

In the classical description, which we owe to Eddington (1925), Vogt (1925) and Sweet (1950), the centrifugal force causes a thermal imbalance in the radiative region of a rotating star, which in turn generates a large- scale meridian circulation whose turn-over time is

$$
t_{E S}=t_{K H}\left(\frac{\Omega^{2} R^{3}}{G M}\right)^{-1}
$$

with $t_{K H}=G M^{2} / R L$ being the Kelvin-Helmholtz time $(M, R$ and $L$ are the mass, radius and luminosity of the star, $\Omega$ its angular velocity, and $G$ the gravitational constant). This time is short enough for early-type stars to prevent them from ever becoming giants. Mestel (1953) resolved this paradox by explaining that the gradient of molecular weight which arises from hydrogen burning tends to choke the circulation in creating adverse $\mu$-currents; according to him, mixing can only occur in very fast rotators, close to the centrifugal break-up.

But this appears in conflict with some observations, which indicate that helium is enhanced by 15 to $20 \%$ in rapid rotators (Herrero et al. 1992).

The weakness of the classical Eddington-Sweet theory is that it postulates a prescribed rotation law, most often uniform rotation. When one 
takes into the advection of angular momentum through the meridian circulation, which keeps modifying the internal rotation, the picture changes radically. Early-type stars lose little angular momentum during their mainsequence phase, as attested by their large rotation velocities. Hence, there is no need to carry this angular momentum from the interior to the surface, and therefore the circulation tends to vanish after an initial adjustment phase. The proof of this was brought by Busse $(1981,1982)$, but it was met with great scepticism, partly because his analysis was not carried to high enough order and therefore produced an asymptotic solution with uniform rotation.

In fact, it turns out that mixing is not fully suppressed, because even in the asymptotic regime predicted by Busse some differential rotation subsists, and gives rise to shear instabilities (Zahn 1992). The turbulence generated by the horizontal shear tends to restore uniform rotation in latitude, and to first approximation the rotation rate depends only on the radial coordinate: $\Omega=\Omega(r)$. The vertical shear $d \Omega / d r$ is stabilized by the (stable) entropy stratification, but only to some extent, because radiative leakage allows for turbulence at small enough scales (cf. Zahn 1974). Thus, a stationary solution is conceivable, in which the angular fluxes carried by the circulation and the turbulent motions cancel each other, and it would allow the star to be mixed.

But, the situation is more intricate, because the star evolves in a nonhomologous way, and angular momentum keeps being redistributed, thus excluding an asymptotic regime. Moreover, the gradient of molecular weight $\nabla_{\mu}=d \ln \mu / d \ln P$ which arises from nuclear burning strongly inhibits the vertical shear instabilities, which play a key role in the mixing near the convective core (Meynet \& Maeder 1996). According to the Richardson criterion, these are suppressed as soon as

$$
\frac{g}{H_{P}} \nabla_{\mu} \geq R i_{c}\left(\frac{r \sin \theta d \Omega}{d r}\right)^{2}
$$

provided no other instability is present $\left(H_{P}\right.$ is the pressure scale-height, $g$ the local gravity, $\theta$ the colatitude and $R i_{c} \approx 1 / 4$ the critical Richardson number). However, we have seen that the horizontal shear produces some turbulence which tends to restore uniform rotation in latitude; although the turbulent motions are highly anisotropic in this stable stratification, they will erode the density fluctuations and thus weaken the buoyancy force. The resulting turbulent transport in the radial direction is characterized by a diffusion coefficient (Talon \& Zahn 1996)

$$
D_{v}=2 R i_{c} \frac{H_{P}}{g} \frac{(r \sin \theta d \Omega / d r)^{2}}{\left(\nabla_{a d}-\nabla\right) /\left(K+D_{h}\right)+\nabla_{\mu} / D_{h}},
$$


where $K$ is the radiative diffusivity and $D_{h}\left(\gg D_{v}\right)$ the horizontal turbulent diffusivity.

Presently, the weak point in the theory is the coefficient $D_{h}$, which we are unable as yet to derive from first principles. It forces us to settle for a substitute, in the form of a parametric relation which may be established as follows. Since the horizontal shear is sustained by the meridian advection of angular momentum, the strength of the turbulence must be related with the amplitude $U$ of the circulation velocity, in order to keep the differential rotation in latitude at some moderate level. This leads to the following prescription, which we shall use in lack of something better (see Zahn 1992):

$$
D_{h}=\frac{r U}{C_{h}}\left[\frac{1}{3} \frac{d \ln \rho r^{2} U}{d \ln r}-\frac{1}{2} \frac{d \ln r^{2} \Omega}{d \ln r}\right]
$$

with $\rho$ being the density and $C_{h}$ a parameter of order 1 .

This description for the transport of matter and angular momentum has been implemented in the Geneva stellar evolution code, and the first calculations which have been performed for the main-sequence evolution of a $9 \mathrm{M}_{\odot}$ star are rather encouraging. They allow indeed for partial mixing, the main effects being observable in the surface abundances of ${ }^{12} \mathrm{C},{ }^{14} \mathrm{~N}$ and ${ }^{16} \mathrm{O}$, accompanied by a slight increase of ${ }^{4} \mathrm{He}$ (Talon et al. 1996; see also the poster presented at this symposium by Talon \& Zahn).

\section{Rotational mixing in late-type stars}

It has been noticed from the start that the depletion of lithium in late-type stars could well be related with their spin-down (see for instance Skumanich 1972). Both the abundance of ${ }^{7} \mathrm{Li}$ and the rotation rate decrease with age; in the Pleiades, where one observes a large spread of these parameters, the fastest rotators display the highest lithium content and the slow rotators are generally lithium depleted (Soderblom et al. 1993).

As was mentioned in $\S 1$, lithium is destroyed at some depth below the convective envelope of these stars, and therefore it has to be carried there by a not too efficient transport process, whose effect is felt in a few $10^{8}$ years: from the age of the Pleiades to that of the Hyades, the lithium abundance decreases roughly by $0.5 \mathrm{dex}$ in a $1 \mathrm{M}_{\odot}$ star.

Likewise, angular momentum must be extracted from the radiative interior of the star, since the torque exerted by the stellar wind is applied on its magnetosphere (Schatzman 1962), and thus on its convective envelope.

It is therefore natural to examine first whether the same physical mechanism may be responsible for both of these transports, and we shall call this possibility rotational mixing of type $I$. 
Two physical processes are known to transport both angular momentum and matter, namely turbulent diffusion and advection through a large-scale circulation. The effect of turbulent mixing has been studied thoroughly at Yale by Endal and Sofia (1978), and more recently by Pinsonneault et al. $(1989,1990)$. In their picture, the magnetic torque slows down the convective envelope, and thereby induces in the radiative interior a differential rotation which is liable to various instabilities, thus leading to turbulent transport. The contribution of the meridian circulation is also included, but for simplicity it too is handled as a diffusive process. As mentioned above, the differential rotation in latitude is smoothed out by horizontal shear instabilities, which permits to consider the angular velocity $\Omega$ as a function of depth only. Its evolution in time is governed by the diffusion equation

$$
\rho \frac{\partial}{\partial t}\left[r^{2} \Omega\right]=\frac{1}{r^{2}} \frac{\partial}{\partial r}\left[\rho \nu_{r o t} r^{4} \frac{\partial \Omega}{\partial r}\right]
$$

with a prescription for the turbulent viscosity $\nu_{\text {rot }}$ inspired by the instabilities which are most likely to occur.

Equation (5) is integrated with a surface boundary condition specifying the loss of angular momentum $d J / d t$ through the wind as a function of the angular velocity, which never departs much from the power law given originally by Skumanich (1972):

$$
\frac{d J}{d t}=-f_{w} \Omega^{3} .
$$

The additional parameter $f_{w}$ involved in that prescription is adjusted so as to yield the present rotation rate.

The transport of lithium (or any other element) is modeled by a similar diffusion equation

$$
\rho \frac{\partial c_{i}}{\partial t}=\dot{c}_{i}+\frac{1}{r^{2}} \frac{\partial}{\partial r}\left\{r^{2} \rho\left[D_{m, 1} c_{i}+\left(D_{m, 2}+f_{c} \nu_{r o t}\right) \frac{\partial c_{i}}{\partial r}\right]\right\}
$$

where $c_{i}$ designates the concentration of the considered species, $\dot{c}_{i}$ its creation or destruction rate through nuclear reactions; the $D_{m, i}$ account for the microscopic diffusion and $f_{c} \nu_{\text {rot }}$ is the turbulent diffusion coefficient.

To yield the observed ${ }^{7} \mathrm{Li}$ abundance, the parameter $f_{c}$ must take a rather small value $(\approx 0.035)$, whereas the turbulent diffusivity and viscosity should be of the same order.

This disparity, which has been pointed out by Law et al. (1984), has no physical reason: it is simply due to the crude treatment of the meridian circulation. When its transport is properly accounted for, the rotation rate obeys an advection/diffusion equation:

$$
\rho \frac{\partial}{\partial t}\left[r^{2} \Omega\right]=\frac{1}{5 r^{2}} \frac{\partial}{\partial r}\left[\rho r^{4} \Omega U\right]+\frac{1}{r^{2}} \frac{\partial}{\partial r}\left[\rho \nu_{v} r^{4} \frac{\partial \Omega}{\partial r}\right]
$$


where $\nu_{v}$ is the turbulent viscosity in the vertical direction, and $U(r)$ the amplitude of the meridian velocity).

The transport of lithium obeys an equation similar to (7)

$$
\rho \frac{\partial c_{i}}{\partial t}=\dot{c}_{i}+\frac{1}{r^{2}} \frac{\partial}{\partial r}\left\{r^{2} \rho\left[D_{m, 1} c_{i}+\left(D_{m, 2}+\nu_{v}+D_{e f f}\right) \frac{\partial c_{i}}{\partial r}\right]\right\}
$$

but it includes an extra diffusivity given by

$$
D_{e f f}=\frac{|r U(r)|^{2}}{30 D_{h}}
$$

where $D_{h}$ is the horizontal diffusivity (4) which we have encountered in $\S 2$. This term represents the contribution of the meridian circulation, which is changed into a vertical diffusion because the turbulent motions associated with the latitudinal shear instabilities tend to erode the chemical composition on horizontal surfaces, hence reducing the advective transport (Chaboyer \& Zahn 1992).

In contrast, the advection of angular momentum is hardly affected by this horizontal homogeneization, because a net momentum flux subsists even when $\Omega$ is constant on horizontal surfaces. We have thus a physical explanation for the different efficiencies characterizing respectively the transport of angular momentum and that of lithium, as observed in the Sun and solar-type stars.

An important property of this type of rotational mixing is that the transport of lithium is tightly linked with that of angular momentum. Therefore, if no other transport process is present, the depletion of lithium is correlated with the loss of angular momentum through the wind. To first approximation, the theory predicts a linear relation between the relative variation of the lithium concentration $c_{L i}$ and that of angular momentum:

$$
\left.\frac{d}{d t} \ln c_{L i} \propto \frac{d}{d t} \ln J\right|_{w i n d},
$$

once the asymptotic regime has been reached (Zahn 1992, 1994).

But, there is an alternate possibility, which we shall call rotational mixing of type II: the extraction of angular momentum may be due to another, more powerful process, which enforces nearly uniform rotation in the radiative interior, whereas the transport of lithium is achieved mainly through the meridian circulation. In that case, the lithium depletion is related directly with the rotation rate:

$$
\frac{d}{d t} \ln c_{L i} \propto-\Omega^{2}
$$


With single stars, one cannot distinguish between these two types of mixing, since during their spin-down the angular velocity decreases with time roughly as $\Omega \propto t^{-1 / 2}$ (Skumanich 1972), and this means that

$$
\frac{d}{d t} \ln J \propto-\Omega^{2} .
$$

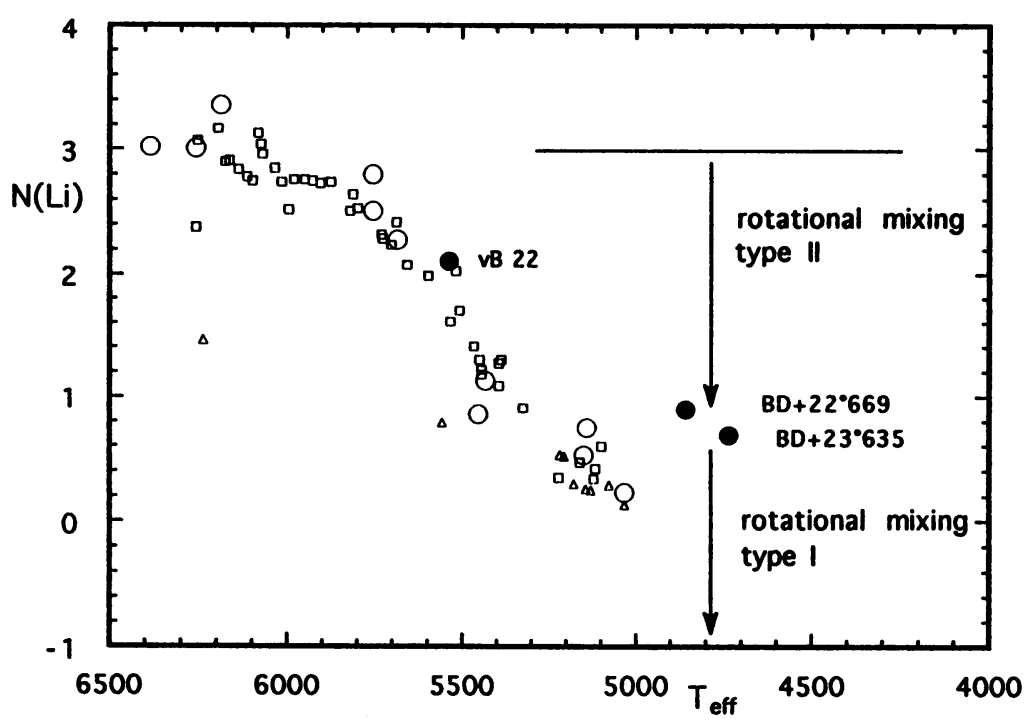

Figure 1. The lithium abundance in the Hyades (on logarithmic scale), as a function of effective temperature (Thorburn et al. 1993). The binaries with known orbital elements are indicated by circles, which are filled for the three tidally-locked binaries (Zahn 1994). Those binaries permit to distinguish between the two types of rotational mixing, as explained in the text.

In tidally locked binaries, however, the angular momentum carried away by the wind is drawn from the orbit, not from the interior of the stars (Ryan \& Deliyannis 1995; Zahn 1994). Therefore, if they are subjected to what we have called rotational mixing of type I, they should deplete less lithium than single stars of the same mass. This is indeed confirmed by the observations, at least to some degree: in the Hyades, the three tidally-locked binaries all show higher lithium abundances than single stars of the same effective temperature (Thorburn et al. 1993).

However, these binaries do not display the original lithium abundance of $\mathrm{N}(\mathrm{Li}) \approx 3$, which indicates that they experience in addition some rotational mixing of type II. The depletion associated with each type of mixing is sketched in Fig. 1. 
The presence of another, more efficient process for the transport of angular momentum is confirmed by the internal rotation of the Sun. If this momentum were transported only by meridian circulation and related turbulent diffusion, as in type I mixing, the solar rotation profile would be rather steep, with the core spinning about 20 times faster than the surface. This was shown already by Pinsonneault et al. (1989), and it has been confirmed in the most recent work by Chaboyer et al. (1995). The calculations we have performed with the improved treatment of the meridian circulation give the same result (cf. the poster by Matias \& Zahn at this symposium).

These predictions are in clear conflict with the solar rotation profile determined by helioseismology, which was presented again during this symposium (see also Brown et al. 1989; Thompson et al. 1996). Below the convective envelope, which is differentially rotating as observed at the surface the rotation appears to be uniform, at least down to a fractional radius of about $r \approx 0.4 R_{\odot}$.

Our conclusion is that the wind-driven circulation certainly plays a role in the transport of angular momentum and chemical species, but that it is insufficient to establish the flat rotation profile observed in the Sun. Therefore, another mechanism must be present, which extracts angular momentum in a more efficient way. We shall examine in turn the two obvious candidates, namely magnetic torquing and wave transport.

\section{Magnetic torquing in solar-type stars}

It has been pointed out long ago by Mestel $(1953,1961,1965)$ and Roxburgh (1963) that even a very weak magnetic field is able to enforce uniform rotation in the radiative interior of a star. The reason is clear: differential rotation winds up any existing meridian (or poloidal) field $\vec{B}_{p}$ into a toroidal component $\vec{B}_{\phi}$, and the resulting Laplace-Lorentz force reacts back on the rotation rate.

The magnetic diffusivity is so small that the toroidal field reaches easily the level where the magnetic torque balances the loss of angular momentum through the wind, which occurs when

$$
r_{c}^{3}<B_{p} B_{\phi}>\left.\approx \frac{d J}{d t}\right|_{w i n d}
$$

at the base of the convection zone $\left(r=r_{c}\right)$. That torque then enforces nearly uniform rotation along the field-lines of the poloidal field $\vec{B}_{p}$, a law first predicted by Ferraro (1937). The rapid evolution towards this state has been described by Mestel, Moss and Tayler (1988), and more recently by Charbonneau and MacGregor (1993), who considered various configurations of the poloidal field. 
However, if the magnetic field is responsible for the extraction of angular momentum from the radiative interior, its poloidal component must be deeply rooted in the radiation zone and it must be connected to the convective envelope. The alternating dynamo field does not satisfy the first condition, since it does not penetrate much into the radiation zone.

A slowly decaying fossil field would comply with both conditions, but it would pose another problem: along each field-line of $\vec{B}_{p}$ the angular velocity would be that of its entry point into the differentially rotating convection zone, which means that the rotation would be non-uniform in the radiative interior, unless all its field-lines converge into the convection zone within a narrow latitude band in each hemisphere. Such a situation cannot be excluded a priori, but no coherent model has been built yet to substantiate it.

Furthermore, the question of the stability of stellar magnetic fields has still not been answered in a satisfactory way, and the subject has not advanced significantly since the excellent review by Spruit (1987). Purely poloidal and purely toroidal fields are known to be dynamically unstable, although rotation may reduce the growth-rate of the instability. Moreover, when radiative damping and magnetic diffusion is taken into account, additional instabilities occur, of the double diffusive type.

We thus conclude that magnetic torquing is a very efficient process to put the radiation zone of the Sun into corotation along the field-lines of the poloidal field, but that the outcome would probably be a differentially rotating interior, contrary to the observations. Furthermore, it remains to be verified whether an internal field of this type would last long enough to play the expected role.

\section{Internal waves in the solar interior}

Gravity waves (also called internal waves) play an important role in the tidal braking of massive binary stars, as was shown by Zahn (1975) and confirmed by Goldreich and Nicholson (1989). But, they have been invoked only recently as a process which could extract angular momentum from the radiative interior of the Sun (Zahn 1990; Schatzman 1993), and the efficiency of this transport has just been evaluated ( Kumar \& Quataert 1996; Zahn, Talon \& Matias 1996).

These waves are characterized by their cyclic frequency $\sigma$ and their horizontal wavenumber $k_{h}$ (or equivalently by their spherical order $\ell$ ), and these determine the vertical wavenumber $k_{v}$ through the dispersion relation

$$
k_{v}^{2}=\left(\frac{N^{2}}{\sigma^{2}}-1\right) k_{h}^{2} \equiv\left(\frac{N^{2}}{\sigma^{2}}-1\right) \frac{\ell(\ell+1)}{r^{2}},
$$


with $N$ being the Brunt-Väisälä frequency $(\sigma<N)$. In a differentially rotating star, the local frequency $\sigma$ is Doppler-shifted through the horizontal motion, and it varies with depth according to:

$$
\sigma(r)=\sigma_{0}-m \Omega(r)
$$

with $\sigma_{0}$ being the frequency in the inertial frame. It is straightforward to evaluate the flux of angular momentum $\mathcal{F}_{J}$ carried by a travelling wave, which is related to the flux of kinetic energy by

$$
\mathcal{F}_{J}=2 \frac{m}{\sigma} \mathcal{F}_{K}
$$

with $m r$ being the azimuthal component of the horizontal wavenumber. In the adiabatic case, the angular momentum is conserved, i.e.

$$
4 \pi r^{2} \mathcal{F}_{J}(r) \equiv \mathcal{L}_{J}=\text { cst }
$$

but when radiative damping is taken into account, the angular momentum "luminosity" decreases as

$$
\mathcal{L}_{J}(r)=\mathcal{L}_{J}\left(r_{c}\right) \exp -\tau(r),
$$

where $\tau$, akin to an optical depth, is given by

$$
\tau(r)=[\ell(\ell+1)]^{3 / 2} \int_{r}^{r_{c}} K \frac{N^{3}}{\sigma^{4}}\left(\frac{N^{2}}{N^{2}-\sigma^{2}}\right)^{\frac{1}{2}} \frac{d r}{r^{3}},
$$

with $r_{c}$ being the coordinate of the top of the radiation zone, $K$ the radiative diffusivity. (The assumption has been made here that the star is homogeneous; for the general case where composition gradients are present, see Zahn et al. 1996). Note that the damping is tremendously enhanced when $\sigma \rightarrow 0$, which in a differentially rotating star arises at the depth where $m \Omega=\sigma(r)-\sigma_{0}$.

It remains to integrate the angular momentum luminosity $\mathcal{L}_{J}(r)$ over the whole spectrum of the internal waves emitted at the base of the convective envelope. We describe here the approach taken by Zahn et al (1996). Following García López and Spruit (1991), we match the pressure fluctuation in the wave with that of the turbulent convection, and allow the convective eddies of spherical order $\ell_{\omega}$ to generate, by stochastic excitation, waves of lower order $\ell$. Furthermore, we assume that the energy spectrum of the convective motions is adequately represented by the Kolmogorov law. This yields the following expression for the flux of internal wave energy at the top of the radiative interior:

$$
\mathcal{F}_{K}\left(r_{c}\right)=\rho_{c} v_{c}^{3} \frac{\omega_{c}}{N_{c}^{2}} \int_{\omega_{c}}^{N_{c}} \frac{d \omega}{\omega}\left(N_{c}^{2}-\omega^{2}\right)^{\frac{1}{2}}\left[\frac{\omega}{\omega_{c}}\right]^{-2} \int_{0}^{\ell} \frac{d \ell}{\ell_{c}}
$$


with $\omega_{c}, \ell_{c}$ and the velocity $v_{c}$ corresponding to the largest convective eddies, and $N_{c}$ being the Brunt-Väisälä frequency, which takes a finite value here due to convective penetration (see Zahn 1991). To derive the flux of angular momentum, we need to know how the energy is distributed over the azimuthal order $m$. We settle for the simplest assumption, which seems to be substantiated by the solar $p$-modes, namely that this distribution is uniform. Making use of (17), of (19) and of (21), we then get the following expression for the angular momentum luminosity:

$$
\mathcal{L}_{J}(r)=4 \pi r^{2} \frac{\rho_{c} v_{c}^{3}}{N_{c} \ell_{c}} \int_{\omega_{c}}^{N_{c}} \frac{d \omega}{\omega}\left(1-\frac{\omega^{2}}{N_{c}^{2}}\right)^{\frac{1}{2}}\left[\frac{\omega}{\omega_{c}}\right]^{-3} \int_{0}^{\ell_{\omega}} \frac{d \ell}{\ell} \int_{-\ell}^{\ell} \exp (-\tau) m d m
$$

Obviously, the damping factor $\exp (-\tau)$ plays a crucial role in this integral: in order to extract angular momentum from the deep interior of the Sun, a wave must be able to reach that far, which requires its spherical order $\ell$ to be as small as possible. A drastic simplification consists in retaining only those waves for which the Sun would be transparent, if their frequency were not Doppler-shifted to zero. For moderate differential rotation, this leads to

$$
\mathcal{L}_{J}(r)=\mathcal{L}_{J}\left(r_{c}\right)-\frac{4 \pi r^{2}}{3} \frac{\rho_{c} v_{c}^{3}}{N_{c} \ell_{c}}\left(\frac{\omega_{c}^{4}}{I}\right) \frac{\Omega(r)-\Omega\left(r_{c}\right)}{\omega_{c}},
$$

with $I(r)$ being the damping integral deduced from (20)

$$
I(r)=\int_{r}^{r_{c}} K N^{3} \frac{d r}{r^{3}} .
$$

If the wave transport were the only present, the angular velocity would evolve according to

$$
\frac{\partial}{\partial t}\left(\rho r^{4} \Omega\right)=\frac{1}{2} \frac{\rho_{c} v_{c}^{3}}{N_{c} \ell_{c}} \frac{\partial}{\partial r}\left[r^{2}\left(\frac{\omega_{c}^{4}}{I}\right) \frac{\left[\Omega(r)-\Omega\left(r_{c}\right)\right]}{\omega_{c}}\right] .
$$

From this equation one readily derives the timescale characterizing this transport process; approximating $\rho_{c} v_{c}^{3}$ by $1 / 10$ of the convective flux, and this flux by $L_{\odot} / 4 \pi r_{c}^{2}$, one obtains an estimate for the synchronization time $t_{\text {sync }}$, with $\bar{\rho}$ being the mean density (see the poster by Zahn et al.):

$$
t_{s y n c} \approx 60 \frac{M_{\odot} R_{\odot}^{2}}{L_{\odot}} \frac{\rho}{\bar{\rho}}\left(\frac{r}{R_{\odot}}\right)^{3}\left(\frac{r_{c}}{R_{\odot}}\right)^{2} N_{c} \omega_{c} \ell_{c}\left(\frac{I}{\omega_{c}^{4}}\right) \approx 10^{7} \text { years. }
$$

The transport efficiency evaluated independently by Kumar and Quataert (1996), using a somewhat different approach, is of the same order. 
Those estimates are obtained with a number of simplifying assumptions, and more work is needed to refine the theory. For instance, the Coriolis force has been ignored in the description of the internal waves; it may well introduce an unbalance between waves of opposite azimuthal order $m$, leading to an extra momentum transport. Moreover, the recipes used to couple the internal waves with the convective motions are rather crude, and these are assumed to obey Kolmogorov's law. Fortunately, the result quoted above is not too sensitive to the slope of the power spectrum.

\section{Conclusion}

We have seen that the rotational mixing due to the meridian circulation and related shear instabilities seems to explain rather well the composition anomalies which are observed in rotating early-type stars (Talon et al. 1996). But, the picture may still be incomplete, and other physical processes may well compete in the transport of angular momentum, as it has become obvious recently for late-type stars. Furthermore, semi-convection contributes also to the mixing in the more massive stars, and it has still not received a satisfactory treatment.

For late-type stars, the situation is also more complex than it was thought before. Although the rotational mixing which we designated by type I is rather successful in modeling the lithium abundance as a function of age (see Pinsonneault et al. 1989, 1990; Chaboyer et al. 1995), it fails to predict the correct internal rotation of the Sun. Having reviewed the few mechanisms which have been proposed so far for the transport of angular momentum in the solar interior, we conclude that the process which seems to prevail, at least in the later stages of evolution, is the transport by internal waves. Its efficiency is so high that it can easily enforce the flat rotation profile revealed by helioseismology (Zahn et al. 1996). This result has been anticipated by Schatzman (1993), and it is confirmed also by the estimates of Kumar and Quataert (1996).

Magnetic torquing is another possibility, although its action depends crucially on the topology of the field, as discussed in $\S 4$. To our best knowledge, no consistent model has been published yet, with realistic boundary conditions at the base of the convection zone and with meridian circulation advecting the decaying poloidal field. But, there is no need to invoke magnetic fields. Most observational facts can be explained by the conjugated action of the rotation-induced meridian circulation and of the gravity waves. Let us sketch what we hold for the most plausible scenario.

During the early phases of the evolution of a solar-type star, while its rotation speed is large, the transport of angular momentum is ensured mainly through the meridian circulation, and the depletion of lithium is 
correlated with the loss of angular momentum by the wind. These processes combine in what we have called the rotational mixing of type I. Their strength scales as the deceleration rate; it thus decreases as the spin-down proceeds. At some point, to be determined by the more detailed calculations which are in progress, the transport of angular momentum by internal waves takes the leading role, and it tends to flatten the rotation profile. This will not stop the meridian circulation, which subsists even when rotation is uniform ( the classical Eddington-Sweet case), but the depletion of lithium is then no longer correlated with the loss of angular momentum: instead, it will scale as $\Omega^{2}$, and we have called this process rotational mixing of type II. Referring back to the tidally-locked binaries in the Hyades which have been discussed above, type I mixing is responsible for the difference in lithium abundance between these binaries and single stars of the same mass, whereas the depletion from the original value $\log \left(\mathrm{N}_{L i}\right) \approx 3$ to the present $\approx$ 1.5 occurs during the later phase, which is dominated by rotational mixing of type II (see Fig. 1).

Further work is required to confirm this scenario, and to adapt it to early-type stars. As top priorities, I would rank a realistic description for the anisotropic shear turbulence which is generated by differential rotation, and an improved treatment for the excitation of internal waves at the boundary of a convection zone. But, it is also very important to verify whether internal waves can contribute directly to the mixing, as it has been suggested by Press (1981), García López and Spruit (1991), and Schatzman (1993, 1996). Finally, we look forward for even tighter observational constraints, and hope that those will be provided soon thanks to helio- and asteroseismic techniques which have been discussed during this symposium.

\section{References}

Biermann, L. (1937) Astron. Nachr., 263, 185

Brown, T.M, Christensen-Dalsgaard, J., Dziembowski, W.A., Goode, P., Gough, D.O. and Morrow, C.A. (1989) $A p J, 343,526$

Busse, F.H. (1981) Geophys. Astrophys. Fluid Dynamics, 17,215

Busse, F.H. (1982) ApJ, 259, 759

Chaboyer, B., Demarque, P. and Pinsonneault, M.H. (1995) ApJ, 441, 865

Charbonneau, P. and MacGregor, K.B. (1993) ApJ, 417, 762

Eddington A.S. (1925) Observatory, 48, 78

Endal A.S. and Sofia, S. (1978) $A p J$, 220, 279

Ferraro, V.C.A. (1937) MNRAS, 97, 458

García López, R.J. and Spruit, H.C. (1991) ApJ, 377, 268

Geiss, J. and Reeves, H. (1972) $A \mathscr{G} A, 18,126$

Giess, D.R. and Lambert, D.L. (1992) $A p J, 387,673$

Goldreich, P. and Nicholson, P.D. (1989) $A p J, 342,1079$

Herbig, G.H. (1965) ApJ, 141, 588

Herrero, A., Kudritzski, R.P., Vilchez, J.M., Kunze, D., Butler, K. and Haser, S. (1992) $A \& A, 261,209$ 
Kumar, P. and Quataert, E.J. (1996) $A p J$, (in press)

Lambert, D.L. (1976) ApJ, 210, 684

Law, W.Y., Knobloch, E. and Spruit, H.C. (1984) Observational Tests of Stellar Evolution

Theory (A. Maeder \& A. Renzini ed.; Reidel)

Lyubimkov, L.S. (1984) Astrofisika, 20, 475

Lyubimkov, L.S. (1991) Evolution of Stars: The Photospheric Abundance Connection (G. Michaud \& A. Tutukov ed.; Kluwer), 125

Meynet, G. and Maeder, A. (1996) $A \mathscr{E} A$, (in press)

Mestel, L. (1953) MNRAS, 113, 716

Mestel, L. (1961) MNRAS, 122, 473

Mestel, L. (1965) Stellar Structure, in Stars and Stellar Systems (G.P. Kuiper \& B.M. Middlehurst; Univ. Chicago Press), 8, 465

Mestel, L., Moss, D. and Taylor, R.J. (1988) MNRAS, 231, 873

Pinsonneault, M.H., Kawaler, S.D., Sofia, S. and Demarque, P. (1989) ApJ, 338, 424

Pinsonneault, M.H., Kawaler, S.D. and Demarque, P. (1990) ApJS, 74, 501

Press, W.H. (1981) ApJ, 245, 286

Roxburgh, I. (1963) MNRAS, 126, 157

Ryan, S.G. and Deliyannis, C.P. (1995) $A p J, 453,819$

Schatzman, E. (1962) Ann. Astrophys., 25, 18

Schatzman, E. (1969) $A \mathscr{G} A, \mathbf{3}, 331$

Schatzman, E. (1993) $A \& A, \mathbf{2 7 9}, 431$

Schatzman, E. (1996) J. Fluid Mech., 322, 355

Skumanich, A. (1972) ApJ, 171, 563

Soderblom, D.R., Burton, F.J., Balachandran, S., Stauffer, J.R., Duncan, D.K., Fedele, S.B. and Hudon, J.D. (1993) $A J$, 106, 1059

Spruit, H.C. (1987) The Internal Solar Angular Velocity (B.R. Durney \& S. Sofia ed.; Reidel) 185

Sweet, P.A. (1950) MNRAS, 110, 548

Talon, S. and Zahn, J.-P. (1996) $A \& A$, (in press)

Talon, S., Zahn, J.-P., Maeder, A. and Meynet, G. (1996) $A \& A$, (in press)

Thompson, M.J., Toomre, J., Anderson, E.R., Antia, H.M., Berthomieu, G., Burtonclay, D., Chitre, S.M., Christensen-Dalsgaard, J., Corbard, T., DeRosa, M., Genovese, C.R., Gough, D.O., Haber, D.A., Harvey, J.W., Hill, F., Howe, R., Korzennik, S.G., Kosovichev, A.G., Leibacher, J.W., Pijpers, F.P., Provost, J., Rhodes Jr., E.J., Schou, J., Sekii, T., Stark, P.B. and Wilson, P.R. (1996) Science, 272, 1300

Thorburn, J.A., Hobbs, L.M., Deliyannis, C.P. and Pinsonneault, M.H. (1993) ApJ, 415, 150

Vogt, H. (1925) Astron. Nachr., 223, 229

Zahn, J.-P. (1974) Stellar Instability and Evolution (P. Ledoux, A. Noels \& R.W. Rogers ed.; Reidel, Dordrecht), 185

Zahn, J.-P. (1975) $A \& A, 41,329$

Zahn, J.-P. (1990) Inside the Sun (G. Berthomieu \& M. Cribier ed.; Kluwer) 425

Zahn, J.-P. (1991) $A \& A$, 252, 179

Zahn, J.-P. (1992) $A \& A$, 265, 115

Zahn, J.-P. (1994) $A \mathscr{E} A$ 288, 829

Zahn, J.-P., Talon, S. and Matias, J. (1996) $A \mathscr{E} A$ (in press) 